\title{
Two-Dimensional Nanostrips of Hydrophobic Copper Tetradecanoate for Making Self-Cleaning Glasses
}

\author{
Subhrangsu Sarkar, ${ }^{1}$ Indrani Mukherjee, ${ }^{2,3}$ Sriparna Chatterjee, ${ }^{2}$ \\ Smita Gohil, ${ }^{1}$ Bimal P. Singh, ${ }^{2}$ and Pushan Ayyub ${ }^{1}$ \\ ${ }^{1}$ Tata Institute of Fundamental Research, 1 Homi Bhabha Road, Mumbai 400005, India \\ ${ }^{2}$ CSIR-Institute of Minerals and Materials Technology, Acharya Vihar, Bhubaneswar 751013, India \\ ${ }^{3}$ National Institute of Technology, Durgapur 713209, India \\ Correspondence should be addressed to Sriparna Chatterjee; sriparna@immt.res.in
}

Received 13 January 2016; Revised 21 February 2016; Accepted 22 February 2016

Academic Editor: Lucia M. Veca

Copyright (C) 2016 Subhrangsu Sarkar et al. This is an open access article distributed under the Creative Commons Attribution License, which permits unrestricted use, distribution, and reproduction in any medium, provided the original work is properly cited.

\begin{abstract}
We report a simple, solution-based technique for coating arbitrary surfaces with thin layers of self-assembled copper tetradecanoate (CTD) nanostrips, resulting in an optically transparent, superhydrophobic coating. The nanostrip-coated surfaces show water contact angles close to $150^{\circ}$ and roll-off angles as small as $2^{\circ}-3^{\circ}$. Importantly, CTD retains its hydrophobic nature even after annealing the self-assembled nanostrips at $200^{\circ} \mathrm{C}$, which does not alter the crystal structure but "melts" the surface microstructure. This clearly indicates that the hydrophobicity in CTD is likely to be intrinsic in nature and not induced by the surface microstructure (as has been suggested earlier). Strong hydrophobicity in CTD over a relatively wide temperature range presumably results from the presence of the long aliphatic (tetradecanoate) chains in its structure. Importantly, the self-assembled copper tetradecanoate nanostrips can be dip-coated on glass to render it hydrophobic and at the same time retain a significant level of transparency over the entire visible region. Such nanostructured thin films may be expected to find applications not only as a self-cleaning glass, but also as a corrosion resistant coating, in gas storage (due to the layered structure), and as an active catalyst because of the visible absorbance.
\end{abstract}

\section{Introduction}

The wetting behavior of a solid surface is known to be controlled not only by the physicochemical properties of the material but also by the exact nature of the surface microstructure [1], usually via the Cassie-Baxter mechanism. In fact, both the contact angle and the roll-off angle (which determines the "stickiness" of the surface) can be independently controlled to a large extent in biomimetically fabricated, hierarchical (2-level) micronanostructures [2-4]. We have earlier shown that even a metallic surface (which is generally strongly hydrophilic) can be made superhydrophobic when it has the appropriate micronanostructure, such as a clustered array of metallic nanorods $[5,6]$. It is clear that the study of hydrophobic surfaces, whether natural or artificial, continues to be an active field of research
$[7,8]$ with direct, large-scale industrial applications as protective coatings with corrosion resistance and antiwetting properties. Materials that are naturally hydrophobic over a wide temperature range are particularly attractive, since they could presumably be rendered superhydrophobic by suitable surface structuring. In this regard, copper tetradecanoate (CTD) appears to be a promising material in which superhydrophobicity has been reported relatively recently. Wang et al. [9] reported the observation of morphologydriven superhydrophobicity in self-assembled microflowerlike CTD structures grown on $\mathrm{Cu}$ substrates. Having the structural formula $\mathrm{Cu}\left[\mathrm{CH}_{3}\left(\mathrm{CH}_{2}\right)_{12} \mathrm{COO}\right]_{2}$, copper carboxylate, more precisely termed copper tetradecanoate, has a hybrid organic-inorganic structure that incorporates a long alkyl chain in addition to the tetradecanoate functional group. CTD is also known for its fungicidal activity [10], 
mediator in substituted alkyne synthesis [11], and electrocatalyst for oxygen reduction reaction [12]. Currently there are several different approaches $[13,14]$ to the preparation of superhydrophobic materials. However, an industrially attractive process is required to be simple, scalable, environmentally compatible (using nontoxic chemicals), chemically and thermally stable, and cost effective $[15,16]$. In view of these considerations, solution-immersion appears to be an appropriate and attractive technique for controlling the wettability of the solid surface. In this work, we have explored the microstructural, thermal, and optical properties of CTD nanostrips coated on glass. Importantly, our contact angle $\left(\theta_{\mathrm{C}}\right)$ measurements on as-prepared and annealed samples (in which the microstructure is smoothened out) appear to show that copper(II) tetradecanoate is intrinsically hydrophobic $\left(\theta_{\mathrm{C}} \approx 140^{\circ}\right.$ and tilt angle $\left.\approx 2^{\circ}-3^{\circ}\right)$. Earlier reports had ascribed the high contact angle to the surface microstructure. For comparison, we also grew the microflower structures on copper substrates treated with a solution of tetradecanoic acid (TDA) and ethanol $[9,17]$. Again, we found only a small decrease in the contact angle on annealing out the microstructure. The intrinsic hydrophobicity of CTD can be attributed to the presence of the long hydrocarbon chain in its molecular structure, which results in low electronic polarizability and hence low critical surface tension. This appears to be the first study of copper tetradecanoate in the form of nanoplatelets (or strips), with interesting application in making transparent self-cleaning glasses. We have used a simple, scalable dip-coating process to produce CTD coated glass slides that are shown to be hydrophobic and transparent in the visible region. Using this technique, self-assembled copper tetradecanoate nanostrips can be coated on any solid surface to render it strongly hydrophobic. Such materials may find applications not only in corrosion resistant coatings, but also as catalyst supports and battery materials.

\section{Materials and Methods}

We employed a modified solution-immersion protocol for depositing nanostrips of copper(II) tetradecanoate, on glass. $\mathrm{Cu}$ powder (Johnson Matthey, 99.9\%) was suspended in $0.01 \mathrm{M}$ ethanolic tetradecanoic acid (Sigma, 99\%), giving a brown suspension that was stirred continuously for $72 \mathrm{~h}$ in a sealed container at room temperature. The as-prepared CTD was centrifuged, thoroughly washed with ethanol, and dried at ambient temperature. The coating solution was made by dispersing about $200 \mathrm{mg}$ CTD powder in $10 \mathrm{~mL}$ ethanol by ultrasonication for $2 \mathrm{~h}$. The CTD films were coated on clean, $2 \mathrm{~cm} \times 2 \mathrm{~cm}$ soda-lime silica glass using a dip coater (SDC 2007C, Apex Instruments), with a withdrawal speed of $10 \mathrm{~cm} / \mathrm{min}$. The coated slides were dried overnight in air. For comparison, microflower-structured CTD was separately grown on a $\mathrm{Cu}$ foil using the previously reported process [9]. Powder X-ray diffraction (XRD) data were recorded using a PANalytical X'pert Pro diffractometer with $\mathrm{CuK} \alpha$ radiation. The microstructure of the CTD samples was studied with a Zeiss Ultra 55 Plus field emission scanning electron microscope (SEM) and a Carl Zeiss LIBRA 120 transmission electron microscope (TEM). Thermogravimetric analysis
(TGA) and differential thermal analysis (DTA) were carried out using STA 449 F1 Jupiter Simultaneous TGA-DSC. Water contact angles were measured (using a Dataphysics OCA15EC instrument) at $25^{\circ} \mathrm{C}$ at different locations on the sample to check for consistency.

\section{Results and Discussion}

Copper(II) tetradecanoate (CTD) nanostrips were successfully synthesized by a one-step solution-immersion process and the thermal stability of the as-prepared nanostrips was investigated up to $500^{\circ} \mathrm{C}$ in an Argon atmosphere using TGDTA with a ramp rate of $10^{\circ} \mathrm{C} \mathrm{min}^{-1}$. The TGA curve in Figure 1(a) shows negligible mass loss up to $240^{\circ} \mathrm{C}$ but there is almost $70 \%$ drop between $260^{\circ} \mathrm{C}$ and $330^{\circ} \mathrm{C}$. Beyond $330^{\circ} \mathrm{C}$ the change in mass is again very small. We can assume that CTD decomposes into the oxides of copper in this temperature interval. In contrast, pure tetradecanoic acid (TDA) starts to decompose by $190^{\circ} \mathrm{C}$ with a $100 \%$ mass loss (Figure 1(b)). CTD nanostrips also undergo a lowtemperature transition that is manifested in the DTA data as a prominent endothermic dip at $120^{\circ} \mathrm{C}$ but does not involve a corresponding mass loss. These observations led us to investigate the morphology and chemical nature of the asprepared CTD nanostrips as well as nanostrips heated to $200^{\circ} \mathrm{C}, 325^{\circ} \mathrm{C}$, and $450^{\circ} \mathrm{C}$. Representative SEM (Figure 1(c)) and TEM (Figure 1(e)) images of a collection of as-deposited CTD nanostrips indicate that their lengths are typically in the 5-10 $\mu \mathrm{m}$ range and widths typically in $200-500 \mathrm{~nm}$ range, giving an overall aspect ratio between 10 and 50. The XRD pattern (overlaid in Figure 1(c)) shows a series of welldefined diffraction lines in the low angle region $\left(<20^{\circ}\right)$ with $d$-spacings in the ratio $1: 1 / 2: 1 / 3 \cdots 1 / n$ that identify them as the (h00) lines of a perfectly layered structure such as CTD [9] with a mean interlayer separation of $3.75( \pm 0.01)$ $\mathrm{nm}$. The SEM image (Figure 1(d)) shows that annealing at $200^{\circ} \mathrm{C}$ completely destroys the basic nanostrip morphology and gives rise to smooth, $\sim 50 \mu \mathrm{m}$ grains. The XRD spectrum of the $200^{\circ} \mathrm{C}$-annealed sample (overlaid) still shows lines due to the (300), (400), and (500) planes, confirming that the layered crystal structure of CTD remained intact, though the high degree of orientational order (in the as-prepared sample) is obviously lost. Also, the sample annealed at $200^{\circ} \mathrm{C}$ still appeared bright green, just as the as-prepared one. These observations lead us to the inescapable conclusion that the DTA transition at $120^{\circ} \mathrm{C}$ represents the "melting" of the CTD nanostrips into comparatively large-sized, fused structures, which are clearly seen in Figure 1(d). The CTD nanostrip sample annealed at $325^{\circ} \mathrm{C}$ was found to be converted into black, equiaxed particles of $\mathrm{CuO}$, shown in Figure 1(f). Such a transformation was already indicated by the thermoanalytical data and confirmed by the XRD data overlaid in Figure 1(f). The loosely aggregated $\mathrm{CuO}$ nanoparticles have a Scherrer diameter of $17 \mathrm{~nm}$. Finally, on annealing the as-prepared CTD nanostrip sample at $450^{\circ} \mathrm{C}$, we observed larger-sized (Scherrer diameter $=35 \mathrm{~nm}) \mathrm{CuO}$ nanoparticles. The sequence of changes that occur on annealing is summarized in Table 1.

Figure 2 shows the Fourier Transform Infrared (FTIR) spectra in the $4000-400 \mathrm{~cm}^{-1}$ range for tetradecanoic acid 


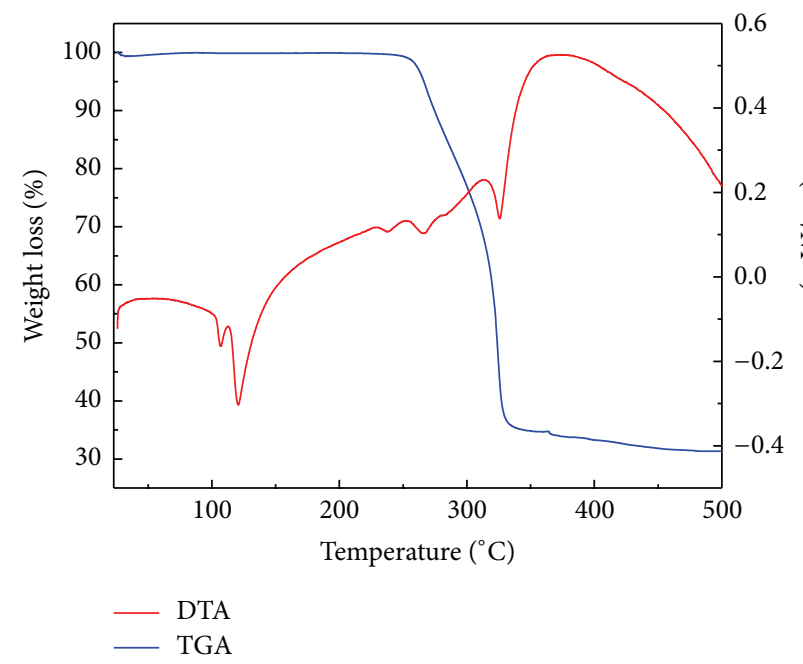

(a)

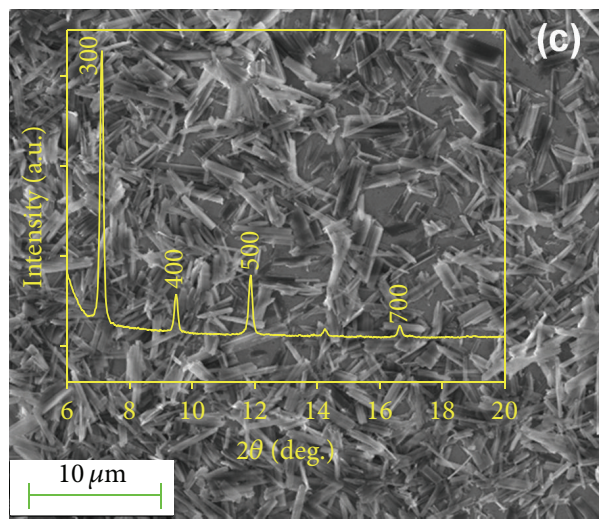

(c)

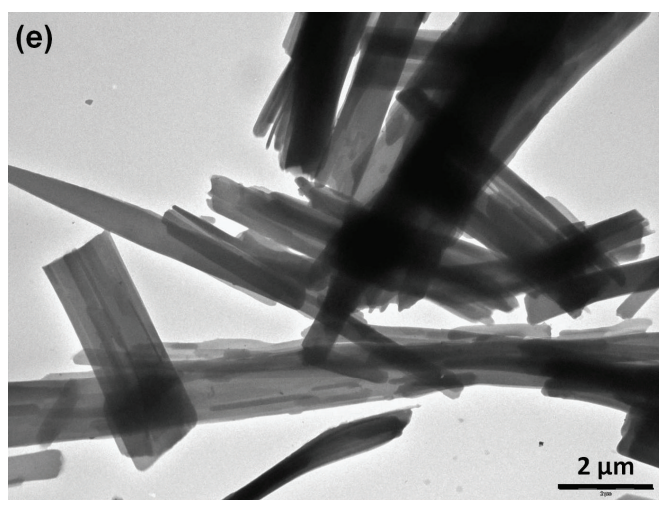

(e)

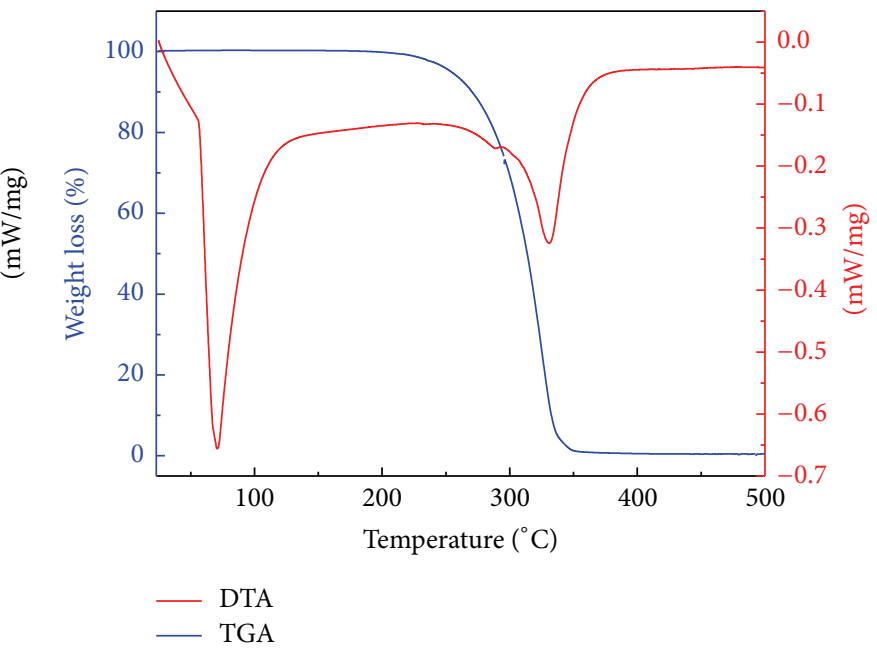

(b)

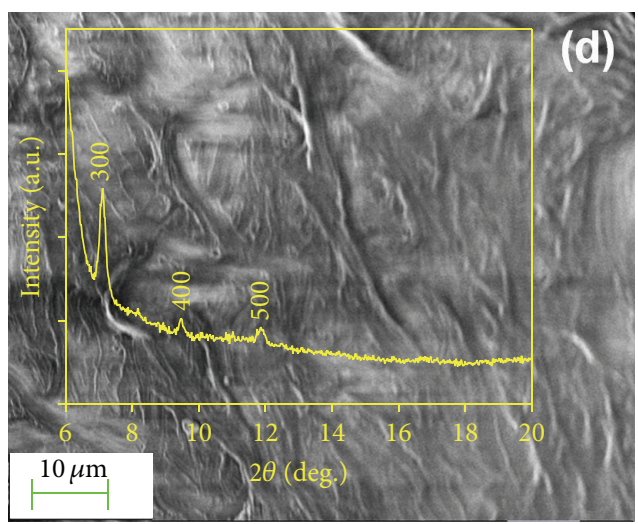

(d)

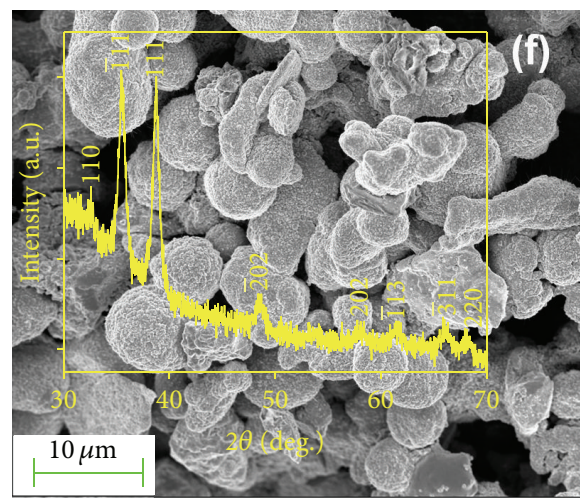

(f)

Figure 1: Thermoanalytical data (TGA and DTA) of (a) copper tetradecanoate nanostrip powder, showing a melting transition at $\approx 130^{\circ} \mathrm{C}$ and decomposition above $\approx 270^{\circ} \mathrm{C}$, and (b) pure tetradecanoic acid. Typical (c) scanning electron micrograph and transmission electron micrograph (e) of copper tetradecanoate nanostrips synthesized from copper powder. SEMs of copper tetradecanoate nanostrips annealed at (d) $200^{\circ} \mathrm{C}$ and (f) $325^{\circ} \mathrm{C}$. The respective X-ray diffractograms are shown overlaid for each sample.

(TDA), as-prepared CTD nanostrips, and CTD nanostrips annealed at $200^{\circ} \mathrm{C}$ (CTD-200). The IR modes of both the as-prepared and annealed samples that were observed at $1727 \mathrm{~cm}^{-1}, 1448 \mathrm{~cm}^{-1}, 1310 \mathrm{~cm}^{-1}$, and $711 \mathrm{~cm}^{-1}$ can be attributed, respectively, to the aldehyde $\mathrm{C}=\mathrm{O}$ stretching and
C-C stretching, C-O stretching of carboxylic acid or ester, and $\mathrm{C}-\mathrm{H}$ bending vibrations. There is no obvious change in the bands representing the methylene and methyl groups that appear in the $2000-3000 \mathrm{~cm}^{-1}$ range. In the case of $\mathrm{n}$ tetradecanoic acid, the free COO band is present at $1702 \mathrm{~cm}^{-1}$ 
TABLE 1: Effect of annealing on the structure and composition of the as-prepared copper tetradecanoate (CTD) nanostrips.

\begin{tabular}{|c|c|c|c|c|}
\hline Sample & $\begin{array}{l}\text { Morphology } \\
\text { (SEM) }\end{array}$ & $\begin{array}{c}\text { Crystal structure } \\
\text { (XRD) }\end{array}$ & $\begin{array}{c}\text { Chemical species } \\
\text { (FTIR) }\end{array}$ & $\begin{array}{l}\text { Nature of } \\
\text { Sample }\end{array}$ \\
\hline $\begin{array}{l}\text { CTD (as prepared) } \\
\text { light green }\end{array}$ & 2-D Nanostrips & $\begin{array}{l}\text { Only }(h 00) \text { lines of } \\
\text { CTD }\end{array}$ & $\begin{array}{c}\text { coordinated COO } \\
\text { band }^{*}\end{array}$ & $\begin{array}{c}\text { CTD nanostrips } \\
5 \mu \mathrm{m} \times 0.2 \mu \mathrm{m} \\
\end{array}$ \\
\hline $\begin{array}{l}\text { CTD-200 (annealed } \\
200^{\circ} \mathrm{C} \text { ), light green }\end{array}$ & $\begin{array}{l}\text { Large, molten, } \\
\text { smooth grains }\end{array}$ & $\begin{array}{l}\text { Only }(h 00) \text { lines of } \\
\text { CTD }\end{array}$ & $\begin{array}{l}\text { coordinated COO } \\
\text { band }^{*}\end{array}$ & $\begin{array}{l}\text { Molten CTD, } \\
\sim 50 \mu \mathrm{m} \text { grains }\end{array}$ \\
\hline $\begin{array}{l}\left.\text { CTD-325 (annealed } 325^{\circ} \mathrm{C}\right) \text {, } \\
\text { black }\end{array}$ & $\begin{array}{l}\text { Isolated spheroidal } \\
\text { particles }\end{array}$ & Monoclinic $\mathrm{CuO}$ & & $\begin{array}{c}\text { Granular } \mathrm{CuO} \\
17 \mathrm{~nm}\end{array}$ \\
\hline $\begin{array}{l}\mathrm{CTD}-450 \text { (annealed } \\
450^{\circ} \mathrm{C} \text { ), black }\end{array}$ & $\begin{array}{c}\text { Isolated, larger, } \\
\text { spheroidal } \\
\text { particles }\end{array}$ & Monoclinic $\mathrm{CuO}$ & & $\begin{array}{c}\text { Granular } \mathrm{CuO} \\
35 \mathrm{~nm}\end{array}$ \\
\hline
\end{tabular}

${ }^{*}$ This IR band is a characteristic feature of copper tetradecanoate (CTD).
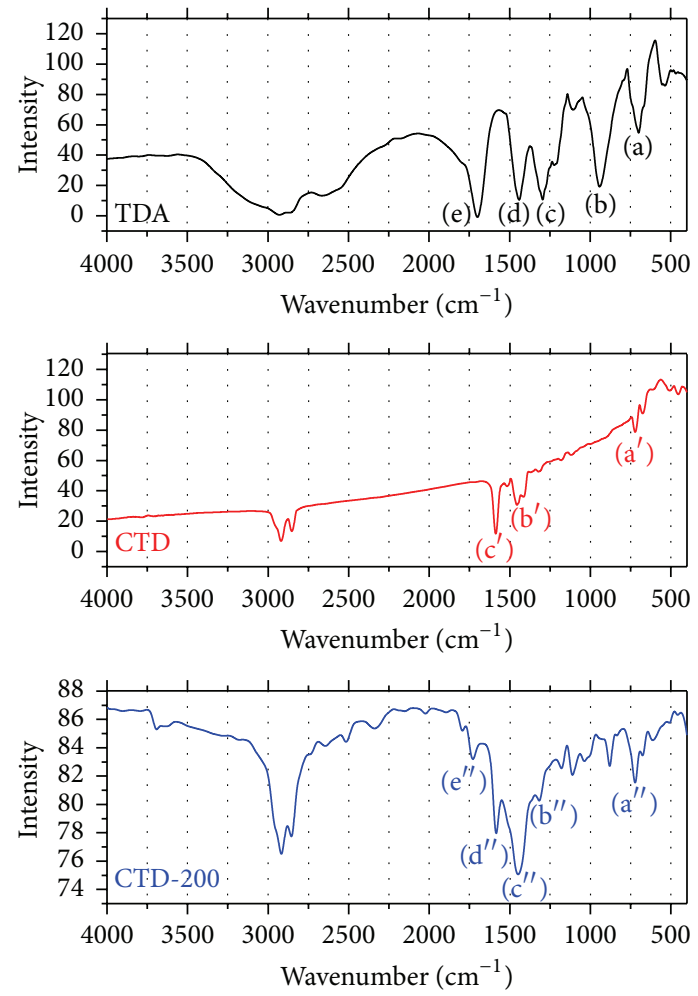

FIGURE 2: FTIR spectra of tetradecanoic acid (TDA), as-synthesized copper tetradecanoate nanostrip powder (CTD), and copper tetradecanoate nanostrips annealed at $200^{\circ} \mathrm{C}$ (CTD-200).

but this band is no longer present in the CTD nanostrips. However, a new band appears at $1587 \mathrm{~cm}^{-1}$ that can be assigned to the coordinated COO band. This band signals the formation of CTD nanostrips. The FTIR spectrum of CTD nanostrips annealed at $200^{\circ} \mathrm{C}$ too showed a band near $1587 \mathrm{~cm}^{-1}$ which is characteristic of the coordinated -COO bond in CTD.

The wettability of the CTD-covered glass slides was investigated by measuring the contact angles for a $0.5 \mu \mathrm{L}$ water droplet as a function of the number of dip-coating cycles. Figure 3 shows the SEM images of the surfaces of a series of glass slides coated with increasing thicknesses of
CTD as well as the respective optical micrographs of the water droplets on these CTD-covered slides. We see that, with an increase in number of coating cycles, the coverage density of the CTD nanostrips on the glass slides becomes greater, as expected. At the same time the water contact angle on glass slides also increases quite markedly (see Table 2). Almost complete coverage with a corresponding contact angle of about $130^{\circ}$ was obtained in 10 cycles. Finally, the sample that was dip-coated for 30 cycles (G30) was fully covered by CTD nanostrips, and this sample also exhibited the largest contact angle $\left(147^{\circ} \pm 2^{\circ}\right)$ as well as an extremely low tilt-off angle $\left(\approx 3^{\circ}\right)$. Since the wettability of a solid surface depends on the surface free energy as well as the nature of the surface microstructure, it is important to ascertain the intrinsic wetting property of a material. We point out that Wang et al. had earlier observed superhydrophobicity in microflower-structured copper tetradecanoate grown on a $\mathrm{Cu}$ plate [9] and ascribed this behavior straightaway to the hierarchical surface microstructure. In the present study, however, we observe strong hydrophobicity even in the nanostrip covered glass slides, which do not appear to possess any obvious hierarchical microstructure. Is copper tetradecanoate therefore intrinsically hydrophobic?

To ensure consistency with earlier data, we prepared CTD in the form of the hierarchical microflowers on a copper plate (Figure 4(a)) following the method of Wang et al. The contact angle and the role-off angle measured for this reference sample (Figure $4(\mathrm{~b})$ ) were $160^{\circ}$ and $2^{\circ}$, respectively, in excellent agreement with the corresponding values reported in [9] $\left(162^{\circ}\right.$ and $\left.2^{\circ}\right)$. Since the nanostrips studied by us exhibit random, somewhat irregular nanostructured surfaces, we may infer that the microflower-like surface structure has a relatively minor role, at best helping to increase the contact angle from $150^{\circ}$ to $160^{\circ}$ owing to the Cassie-Baxter mechanism. To understand the effect of the surface nanostructure more precisely, we annealed both the microflower structured CTD sample (MF) and CTD nanostrip covered glass slide, which was dip-coated 30 times (G30), in an inert atmosphere at $200^{\circ} \mathrm{C}$. As seen earlier, this process results in complete "melting" of the surface microstructure, without any change in composition. In both cases, after annealing, the surfaces were seen to become almost unstructured and much smoother than their as-prepared counterparts. We do 

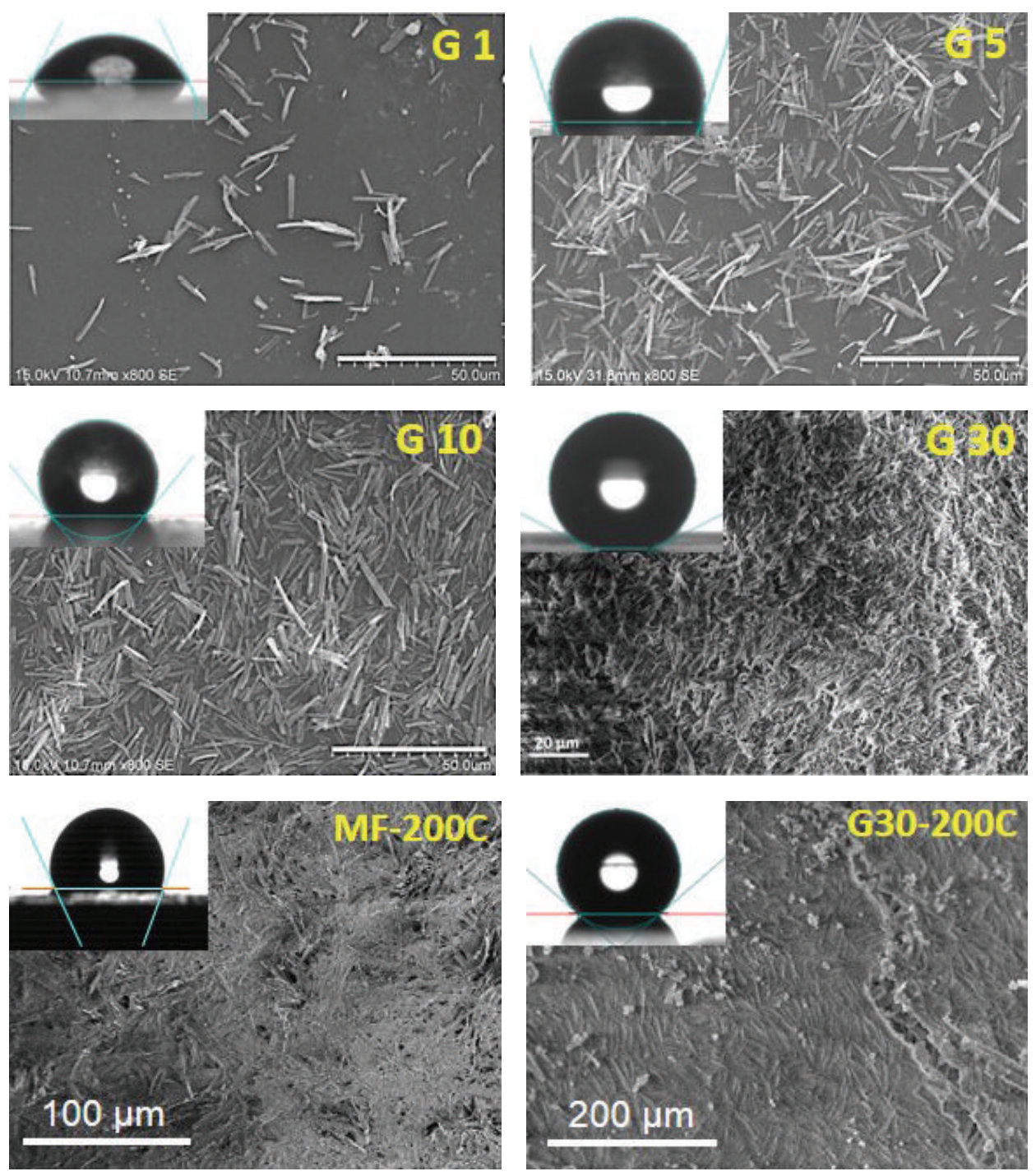

FIGURE 3: Scanning electron micrograph of copper tetradecanoate nanostrip-covered glass slides produced by dip-coating once (G1), five times (G5), ten times (G10), and thirty times (G30). SEMs of postannealed $\left(200^{\circ} \mathrm{C}\right)$ microflower-structured copper tetradecanoate (MF$\left.200^{\circ} \mathrm{C}\right)$ and 30 times dip-coated nanostrips of copper tetradecanoate $\left(\mathrm{G} 30-200^{\circ} \mathrm{C}\right)$ are also shown. Insets show the contact angle for water droplets on these surfaces.

not expect appreciable Cassie-Baxter enhancement in the contact angle from such surfaces. Nevertheless, in both cases the annealed samples retain to a large degree their strongly hydrophobic nature, the contact angle getting reduced merely by $10^{\circ}$ for the microflower sample and $17^{\circ}$ for the nanostrip sample (Figure 3 and Table 2). Our data therefore indicate that CTD is inherently hydrophobic, probably due to the presence of the long aliphatic chain in its molecular structure.

Figure 5(a) shows the optical transmittance spectra of the CTD nanostrip-covered glass slides as a function of the number of dip-coating cycles. All the samples are highly transmitting in the visible range. Even after ten coating cycles of CTD, the glass slide was found to transmit more than $85 \%$ at $550 \mathrm{~nm}$. Figure 5(b) summarizes the correlation between the water contact angle and the transmittance of glass slide at $550 \mathrm{~nm}$ with the number of dipping cycles. According to the data available, the glass slide subjected to 15 dip-coating cycles shows a reasonably high water contact angle of about $136^{\circ}$ combined with a reasonably high transmittance of $80 \%$ at $550 \mathrm{~nm}$. Increasing the coating thickness further increases the contact angle but at the cost of the transmittance going down. This suggests that the CTD-covered glass slides can be potentially used as transparent self-cleaning glasses.

\section{Conclusions}

Earlier studies have attributed the observed superhydrophobicity in copper tetradecanoate to a self-assembled, hierarchical microflower-like structure grown on copper plates. We, however, show that even randomly oriented, unstructured nanostrips of copper tetradecanoate are almost as strongly hydrophobic, with a contact angle of $150^{\circ}$ and roll-off angle 
TABLE 2: Measured values of the contact angle and roll-off angle for a water droplet on glass substrates coated with copper tetradecanoate (CTD) in the form of randomly aligned nanostrips (samples G1 to G30) and a microflowerlike surface structure (MF). Contact angles for two samples (G30 and MF) after annealing at $200^{\circ} \mathrm{C}\left(\mathrm{G} 30-200^{\circ} \mathrm{C}\right.$ and $\mathrm{MF}-200^{\circ} \mathrm{C}$, resp.) are also shown.

\begin{tabular}{|c|c|c|c|c|}
\hline Sample ID & Sample description & Dipping cycles & Contact angle & $\begin{array}{c}\text { Roll-off } \\
\text { angle }\end{array}$ \\
\hline G1 & Glass slide dip-coated by CTD nanostrips & 1 & $60.2^{\circ} \pm 3.5^{\circ}$ & - \\
\hline G2 & Glass slide dip-coated by CTD nanostrips & 2 & $60.6^{\circ} \pm 3.7^{\circ}$ & - \\
\hline G3 & Glass slide dip-coated by CTD nanostrips & 3 & $91.3^{\circ} \pm 4^{\circ}$ & - \\
\hline G5 & Glass slide dip-coated by CTD nanostrips & 5 & $110.5^{\circ} \pm 3.5^{\circ}$ & - \\
\hline G7 & Glass slide dip-coated by CTD nanostrips & 7 & $115.9 \pm 3.6^{\circ}$ & - \\
\hline G10 & Glass slide dip-coated by CTD nanostrips & 10 & $129.1^{\circ} \pm 4^{\circ}$ & - \\
\hline G15 & Glass slide dip-coated by CTD nanostrips & 15 & $136.1^{\circ} \pm 3^{\circ}$ & - \\
\hline G20 & Glass slide dip-coated by CTD nanostrips & 20 & $140.4^{\circ} \pm 2^{\circ}$ & - \\
\hline G30 & Glass slide dip-coated by CTD nanostrips & 30 & $147^{\circ} \pm 2^{\circ}$ & $\approx 3^{\circ}$ \\
\hline $\mathrm{G} 30-200^{\circ} \mathrm{C}$ & "G30" annealed at $200^{\circ} \mathrm{C}$ & - & $130^{\circ} \pm 4^{\circ}$ & - \\
\hline MF & CTD microflower on $\mathrm{Cu}$ plate & - & $160^{\circ} \pm 2^{\circ}$ & $\approx 2^{\circ}$ \\
\hline $\mathrm{MF}-200^{\circ} \mathrm{C}$ & "MF" annealed at $200^{\circ} \mathrm{C}$ & - & $150^{\circ} \pm 2^{\circ}$ & $\approx 3^{\circ}$ \\
\hline
\end{tabular}

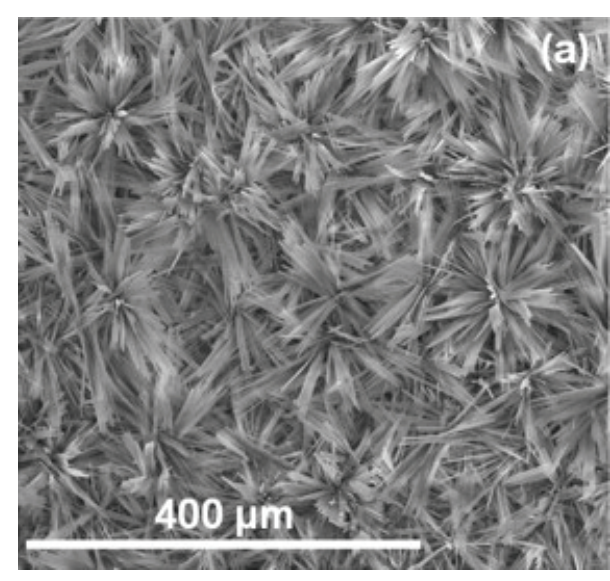

(a)

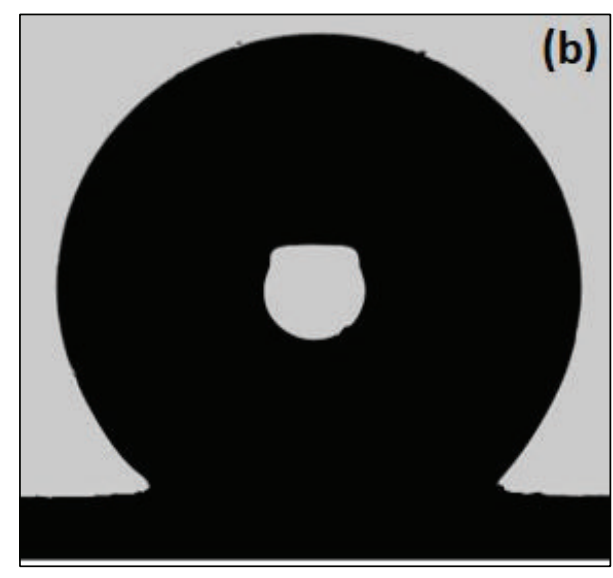

(b)

FIGURE 4: (a) Scanning electron micrograph of copper tetradecanoate microflowers (MF) grown on a Cu plate and (b) optical micrograph showing the contact angle of a water droplet on the above sample.

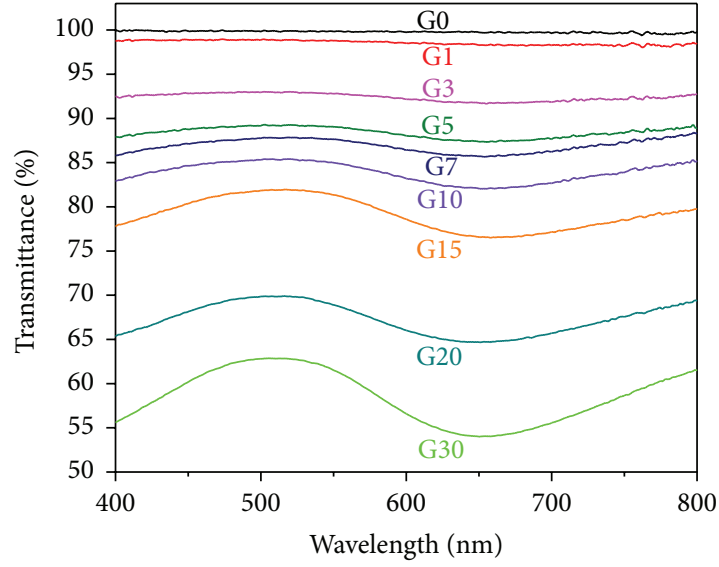

(a)

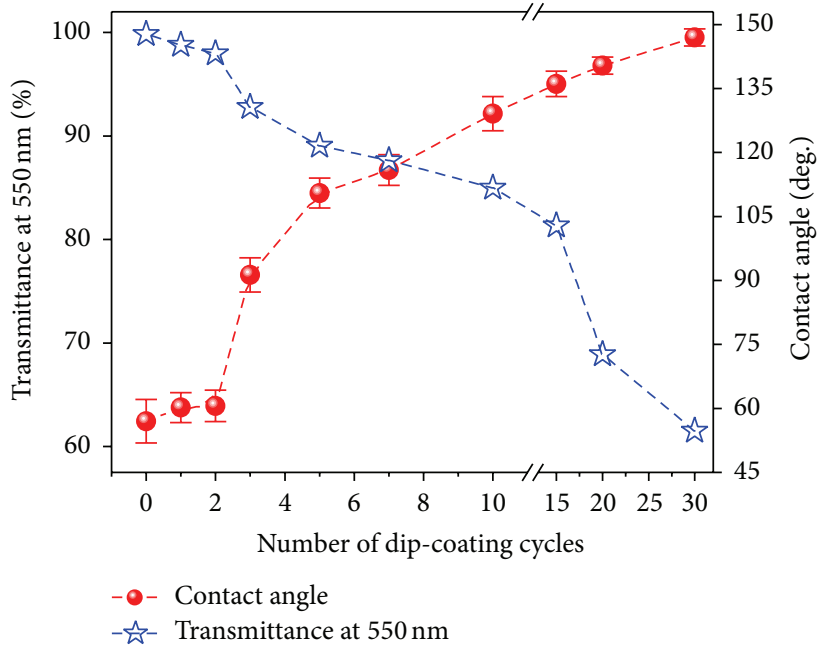

(b)

FIGURE 5: (a) Optical transmittance spectra of nanostrip covered glass slides for various dip-coating cycles (see Table 1 for details). (b) Summary plot showing the dependence of the optical transmittance (at $550 \mathrm{~nm}$ ) and the water contact angle on the number of dip-coating cycles. 
as small as $3^{\circ}$. We further show that, even after "melting" the surface microstructure of both the microflowers and the nanostrips by annealing at $200^{\circ} \mathrm{C}$, there is only a small decrease in the contact angle. Note that annealing at $200^{\circ} \mathrm{C}$ affects neither the chemical composition nor the crystal structure of copper tetradecanoate. This clearly indicates that copper tetradecanoate is naturally hydrophobic, and the surface microstructure only enhances the contact angle by further $10-15^{\circ}$. Our study has a significant functional aspect: owing to the simplicity of the synthesis technique (dipcoating) employed, the self-assembled copper tetradecanoate nanostrips can be coated on any solid surface to render it superhydrophobic. In particular, glass coated in this fashion retains a large part of its optical transparency over the entire visible range. In addition, this material may be expected to find applications in areas such as corrosion coating, catalyst support, and battery material.

\section{Competing Interests}

The authors declare that they have no competing interests.

\section{Acknowledgments}

The authors thank Mr. L. C. Borde for use of the cryoTEM Facility at TIFR and Professor D. Khushalani for several valuable suggestions. S. Chatterjee and I. Mukherjee acknowledge DST, Govt. of India (Grant no. IFA12-CH-65), for financial assistance and thank Professor B. K. Mishra, Director of IMMT, Bhubaneswar, for providing support for this work.

\section{References}

[1] P. G. de Gennes, F. Brochard-Wyart, and D. Quéré, Capillarity and Wetting Phenomena: Drops, Bubbles, Pearls, Waves, Springer, New York, NY, USA, 2003.

[2] B. Bhushan and E. K. Her, "Fabrication of superhydrophobic surfaces with high and low adhesion inspired from rose petal," Langmuir, vol. 26, no. 11, pp. 8207-8217, 2010.

[3] M. Nosonovsky and B. Bhushan, Green Tribology, Green Energy and Technology, Springer, Berlin, Germany, 2012.

[4] I. Chakraborty, N. Singh, S. Gohil, S. Ghosh, and P. Ayyub, "Clustered copper nanorod arrays: a new class of adhesive hydrophobic materials," Soft Matter, vol. 9, no. 48, pp. 11513$11520,2013$.

[5] P. Bhattacharya, S. Gohil, J. Mazher, S. Ghosh, and P. Ayyub, "Universal, geometry-driven hydrophobic behaviour of bare metal nanowire clusters," Nanotechnology, vol. 19, no. 7, Article ID 075709, 2008.

[6] P. Ayyub, "Aligned nanorod arrays: additive and emergent properties," Journal of Cluster Science, vol. 20, no. 2, pp. 429451, 2009.

[7] A. K. Behera, P. Das, I. Thakur, S. Chatterjee, and S. Chatterjee, “Temporal wetting property of 'Micro' versus 'Nano' rods of $\mathrm{ZnO}$ grown using the pressure dependent aqueous solution method," New Journal of Chemistry, vol. 39, no. 11, pp. 89938998, 2015.
[8] S. Dhal, S. Chatterjee, U. Manju et al., "Adhesive hydrophobicity of $\mathrm{Cu}_{2} \mathrm{O}$ nano-coloumnar array induced by nitrogen ion irradiation," Soft Matter, vol. 11, no. 47, pp. 9211-9217, 2015.

[9] S. Wang, L. Feng, and L. Jiang, "One-step solution-immersion process for the fabrication of stable bionic superhydrophobic surfaces," Advanced Materials, vol. 18, no. 6, pp. 767-770, 2006.

[10] A. Golobič, L. Ožbolt, F. Pohleven, I. Leban, and P. Šegedin, "Synthesis, characterization, crystal structures and fungicidal activity of some copper(II) carboxylates with 3-hydroxypyridine," Acta Chimica Slovenica, vol. 53, no. 3, pp. 238-244, 2006.

[11] C. Savarin, J. Srogl, and L. S. Liebeskind, "Substituted alkyne synthesis under nonbasic conditions: copper carboxylatemediated, palladium-catalyzed thioalkyne-boronic acid crosscoupling," Organic Letters, vol. 3, no. 1, pp. 91-93, 2001.

[12] I. Mukherjee, A. K. Samantara, S. Ratha, B. P. Singh, B. K. Jena, and S. Chatterjee, "A facile approach for the synthesis of copper(II) myristate strips and their electrochemical activity towards the oxygen reduction reaction ," RSC Advances, vol. 6, no. 19, pp. 15599-15604, 2016.

[13] J. Xi, L. Feng, and L. Jiang, "A general approach for fabrication of superhydrophobic and superamphiphobic surfaces," Applied Physics Letters, vol. 92, no. 5, Article ID 053102, 2008.

[14] Z. Chen, L. Hao, and C. Chen, "Simultaneous fabrication of superhydrophobic coatings on cathodic and anodic copper surfaces with micro/nano- structures," ECS Electrochemistry Letters, vol. 1, no. 4, pp. D21-D23, 2012.

[15] H. Ogihara, J. Okagaki, and T. Saji, "Facile fabrication of superhydrophobic films by electrophoretic deposition of hydrophobic particles," Chemistry Letters, vol. 38, no. 2, pp. 132-133, 2009.

[16] Y. S. Joung and C. R. Buie, "Electrophoretic deposition of unstable colloidal suspensions for superhydrophobic surfaces," Langmuir, vol. 27, no. 7, pp. 4156-4163, 2011.

[17] T. Liu, Y. Yin, S. Chen, X. Chang, and S. Cheng, "Superhydrophobic surfaces improve corrosion resistance of copper in seawater," Electrochimica Acta, vol. 52, no. 11, pp. 3709-3713, 2007. 

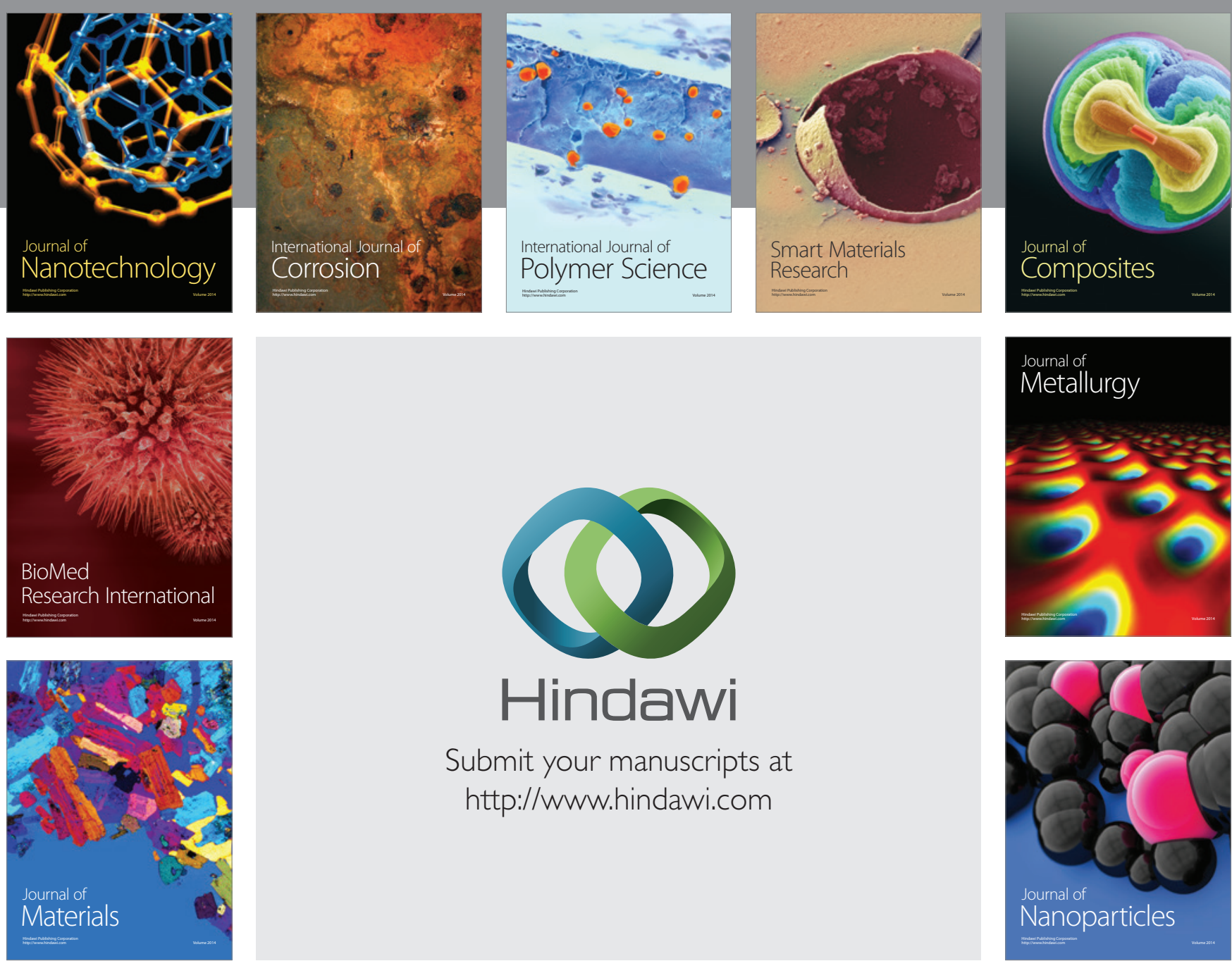

\section{Hindawi}

Submit your manuscripts at

http://www.hindawi.com

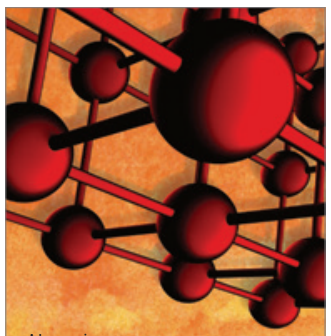

Materials Science and Engineering
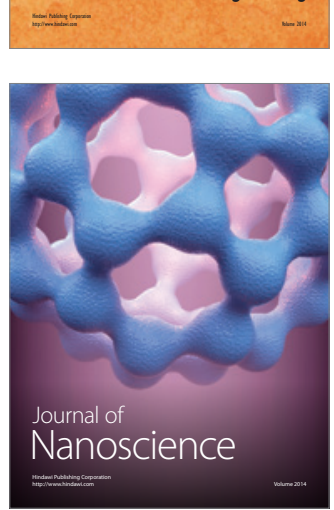
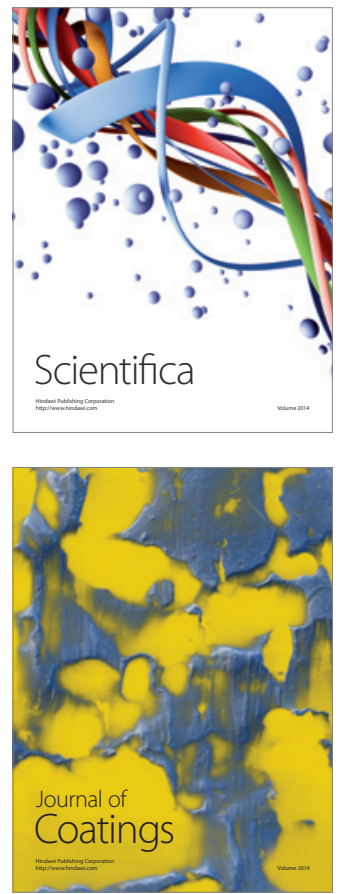
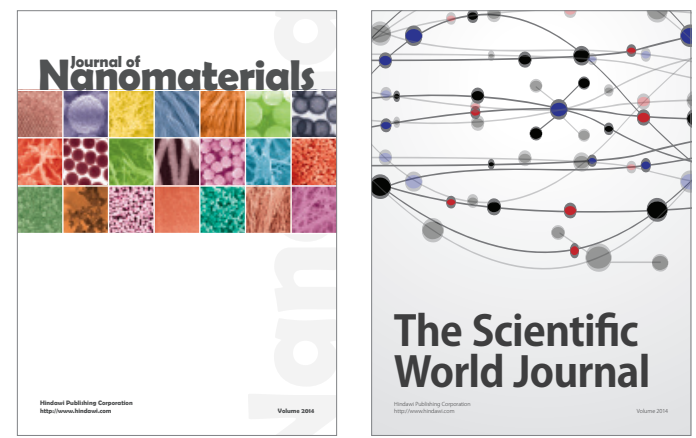

The Scientific World Journal
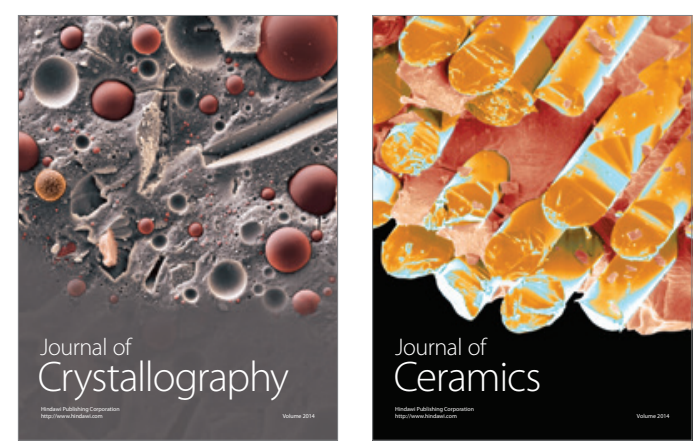
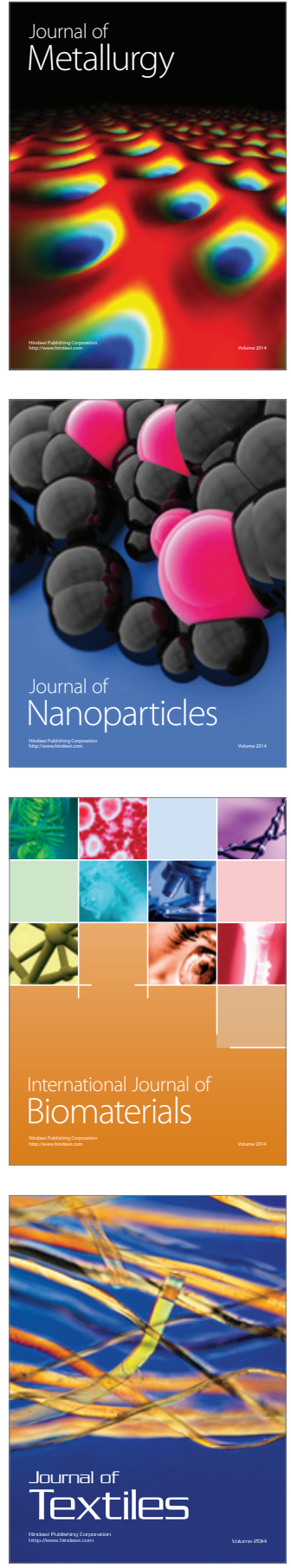